\title{
Behavior of Hydroxide Ions in Vicinity of Self-Assembled Monolayers of Alkanethiols on Metals*
}

\author{
Tomohiro Hayashi ${ }^{\dagger}$ \\ Department of Electronic Chemistry, Interdisciplinary Graduate School of Science and Engineering, \\ Tokyo Institute of Technology, 4259 Nagatsuta-cho, \\ Midori-ku, Yokohama, Kanagawa 226-8502, Japan, and \\ Flucto-Order Functions Asian Collaboration Team, Advanced Science Institute, \\ RIKEN, 2-1 Hirosawa, Wako, Saitama 351-0198, Japan, \\ Yusaku Tanaka and Hiroaki Usukura \\ Department of Electronic Chemistry, Interdisciplinary Graduate School of Science and Engineering, \\ Tokyo Institute of Technology, 4259 Nagatsuta-cho, \\ Midori-ku, Yokohama, Kanagawa 226-8502, Japan, \\ Masahiko Hara \\ Department of Electronic Chemistry, Interdisciplinary Graduate School of Science and Engineering, \\ Tokyo Institute of Technology, 4259 Nagatsuta-cho, \\ Midori-ku, Yokohama, Kanagawa 226-8502, Japan, and \\ Flucto-Order Functions Asian Collaboration Team, Advanced Science Institute, \\ RIKEN, 2-1 Hirosawa, Wako, Saitama 351-0198, Japan
}

(Received 10 November 2008; Accepted 24 February 2009; Published 9 May 2009)

\begin{abstract}
Interactions between self-assembled monolayers (SAMs) with various non-dissociative terminal groups were explored with an atomic force microscope (AFM). In the pure water, hydrophobic $\mathrm{CH}_{3}$-terminated SAMs showed longrange repulsion $(>50 \mathrm{~nm}$ ) indicating that hydroxide ions are concentrated at the water-SAM interface. On the other hand, hydrophilic $\mathrm{OH}$-terminated SAMs exhibited only short-range attraction and no repulsion originated from ions accumulated at the SAM-water interface. The interaction between SAMs of oligo(ethylene glycol)-terminated alkanethiols strongly depend on the substrate ( $\mathrm{Au}$ and $\mathrm{Ag}$ ). That is, the hydroxide ions were concentrated near the Au-supported SAM, whereas no accumulation of the ions near the Ag-supported SAM was observed. Our results demonstrated that not only the terminal groups but also the density of the molecules constituting the SAM critically govern the redistributions of ions at the SAM-water interfaces. [DOI: 10.1380/ejssnt.2009.601]
\end{abstract}

Keywords: Atomic force microscopy; Water; Solid-liquid interfaces; Self-assembly; Biological aspects of nano-structures

\section{INTRODUCTION}

The interfacial behavior of ions at various interfaces has been an important topic in colloid and interface science. The interfacial ions induce the strong electrostatic interaction with other charged or polar groups and the interaction is responsible for the stability of colloids, the fusion of micelles and other biological processes [1]. In this field, the aggregation of ions, especially hydroxide or hydronium ions, to surfaces with non-dissociative functional groups has been a matter of intense debate, since the accumulation of the ions from bulk water to the surface-water interfaces sounds counterintuitive and seems unfavorable from the standpoint of general thermodynamics.

The accumulation of hydroxide ions has been investigated for various interfaces such as water-oil [2], waterpolymer [3], and water-gas interfaces $[4,5]$. Although the results of these experiments clearly suggested that the substantial negative charges were accumulated at the interfaces, the mechanism underlying this phenomenon has not been clear. On the basis of the results of molecular dy-

\footnotetext{
* This paper was presented at International Symposium on Surface Science and Nanotechnology (ISSS-5), Waseda University, Japan, 9-13 November, 2008.

${ }^{\dagger}$ Corresponding author: hayashi@echem.titech.ac.jp
}

namics simulations, Zangi et al. reported that the driving force for the accumulation is the preferential orientation of interfacial waters, which induces an electrical potential gradient [6]. This idea was further refined by Vácha et $a l$. , who concluded that the thermal motion of the surface atoms significantly affects the structure of the interfacial water and the accumulation of hydroxide ions at the interface [7]. Despite several informative reports based on the results of computer simulations, there have been few investigation into the interfacial ions using substrates with well-defined structures and chemical or physical surface properties [8-10].

Self-assembled monolayers (SAMs) of alkanethiols on metal surfaces are one of the ideal organic model substrates with which to study this issue, because the SAMs feature highly ordered and well-defined structures and enable us to relate the molecular-level structures of substrates to the interfacial behavior of the ions $[11,12]$. To investigate that behavior, we used an atomic force microscope (AFM) to measure the force operating between the probe and the substrate as a function of the separation between them with subnanometer accuracy. In this work, we focus on the electrostatic interactions originated from the ions adsorbed on typical hydrophobic and hydrophilic SAMs and SAMs of oligo (ethylene glycol)terminated alkanethiols, which attract much interest due to their protein-resistance abilities $[13,14]$. On the basis of the analysis of the interactive forces, we discuss 
the correlations among the interfacial behavior of ions, surface properties, and the driving forces behind the ion accumulation.

\section{EXPERIMENTAL}

The AFM system used in this study was the commercially available MFP-3D (Asylum Research, Inc., Santa Barbara, CA, USA). We employed a so-called colloid probe that has a silica sphere $(4 \mu \mathrm{m}$ in diameter $)$ glued at the end of a V-shape tip-less cantilever made of $\mathrm{Si}_{3} \mathrm{~N}_{4}$ (the nominal spring constant is $0.06 \mathrm{~N} / \mathrm{m}$ ). The spring constant of each cantilever was calibrated by measuring its thermal fluctuations [15]. In the force curve measurements, the loading force after the contact and the loading rate of the tip were fixed at $250 \mathrm{pN}$ and $600 \mathrm{~nm} / \mathrm{sec}$, respectively.

The probes were cleaned by ultraviolet (UV)-ozone exposure for 15 min just prior to the evaporation of metals to remove any organic contaminants adsorbed on the tip surface. The gold and silver films on the colloid probes were deposited after the evaporation of Ti with a thickness of $2 \mathrm{~nm}$ to promote the adhesion of the films on the probe surfaces. The Au and Ag substrates on mica were prepared by evaporating these metals in vacuum (below $4 \times 10^{-4} \mathrm{~Pa}$ ). The thicknesses of the metal films were 50 $\mathrm{nm}$ on the colloid probes and $100 \mathrm{~nm}$ on the mica substrates, respectively.

Four kinds of alkanethiols with different terminal groups were used. They were n-octanethiol, 11-mercapto1-undecanol, 11-Amino-1-undecanethiol, hydrochloride, and (1-Mercaptoundec-11-yl)tri(ethylene glycol). (Hereafter, the SAMs prepared with these thiols are denoted as $\mathrm{CH}_{3}$-SAM, OH-SAM, $\mathrm{NH}_{2}$-SAM and OEG-SAM, respectively) The $\mathrm{CH}_{3}$-SAM and $\mathrm{OH}-\mathrm{SAM}$ are prototypical examples of hydrophobic and hydrophilic organic surfaces, respectively. The OEG-SAM is an example of a bioinert monolayer, and its protein resistance has been extensively investigated so far [16]. The SAMs were prepared by immersing the substrates and the colloid probes into ethanol solution containing corresponding thiol molecules at a concentration of $1 \mathrm{mM}$ for 24 hours.

To estimate the surface charge density of the SAMs, we performed the fitting of the curves based on the Derjaguin, Landau, Verwey and Overbeek (DLVO) theory [17]. In the case of interaction between a sphere with a diameter of $R$ and a flat surface, the electrostatic doublelayer force interacting between them at a larger separation $(>10 \mathrm{~nm})$ can be expressed as:

$$
F_{\text {electrostatic }}(D)=\frac{4 \pi R \sigma_{S} \sigma_{T} \lambda_{D}}{\epsilon_{0}} \exp \left(-D / \lambda_{D}\right)
$$

where $D$ is the separation between the probe and surface, $\lambda_{D}$ is the Debye decay length and $\sigma_{S}$ and $\sigma_{T}$ are the net charge densities of the surface and probe (in the fittings of this work, these were assumed to be identical) [18].

\section{RESULTS AND DISCUSSION}

As shown in Fig. 1, an attraction between the $\mathrm{CH}_{3}$ $\mathrm{SAM}$ in air was observed at a distance under $20 \mathrm{~nm}$. We

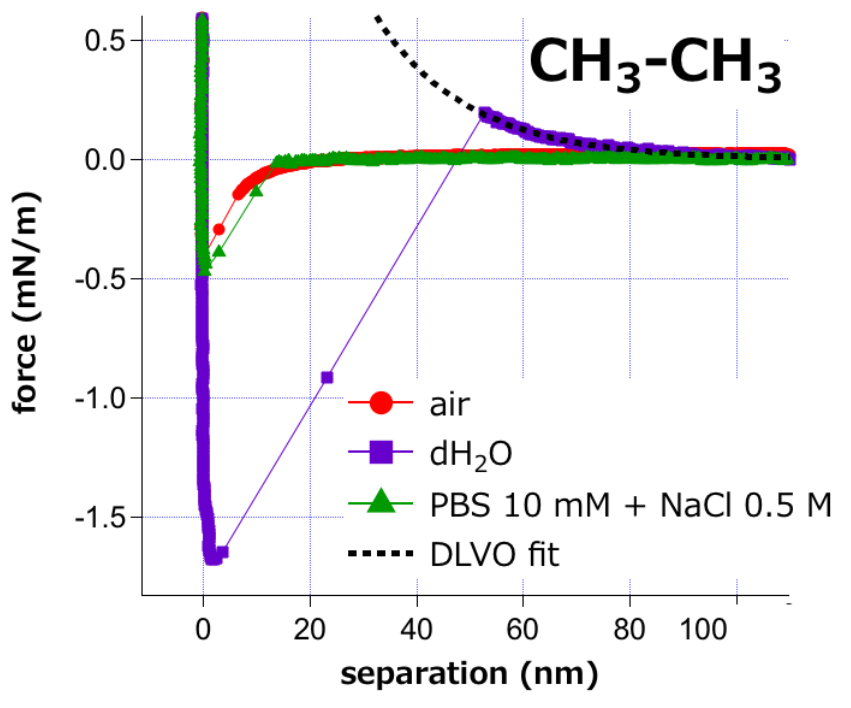

FIG. 1: Force vs distance curves recorded on the approach of a $\mathrm{CH}_{3}$-SAM probe to a $\mathrm{CH}_{3}$-SAM substrate in air, pure water, and buffer solution (PBS buffur $\mathrm{pH} 7.4,10 \mathrm{mM}$ containing $\mathrm{NaCl}$ at a concentration of $0.6 \mathrm{M}$ ) The results of the DLVO fitting was indicated with a dashed line.

identify this as a van der Waals attraction, since there seemed to be no adsorbed water layer, which would have induced a strong attractive capillary force in the vicinity of hydrophobic surfaces [19]. In pure water, a longrange repulsion followed by a jump into contact was observed. The sharp jump-in, which was not observed in air, indicates that the gradient of the attractive force was larger than the spring constant of the cantilever. According to previous reports, the origin of the jump-in can be attributed to capillary force due to the capillary evaporation of water confined between hydrophobic surfaces [20-22]. Comparing the curves measured in pure water to those measured in buffer solution, the disappearance of repulsion with an increase in the ion concentration indicates that this repulsion has an electrostatic origin. To elucidate the origin of the charge, we investigated the interaction between the $\mathrm{CH}_{3}-\mathrm{SAM}$ and $\mathrm{NH}_{2}-\mathrm{SAM}$, which is positively charged in pure water. We found that the attractive force between them (data not shown). This result indicates that the $\mathrm{CH}_{3}-\mathrm{SAM}$ bears negative charges in pure water. TABLE I summarizes the results of the fitting of curves measured in pure water based on the DLVO theory. Chan et al. and Schweiss et al. reported that the SAMs of alkanethiols and hydrophobic polymers were substantially negatively charged due to the adsorbed hydroxide ions and their isoelectric points are around 4.6 $[23,24]$. The estimated surface excess charge density of the $\mathrm{CH}_{3}$-SAM $\left(1.7 \times 10^{-2} \mathrm{e} / \mathrm{nm}^{2}\right)$ was qualitatively comparable to their results $\left(3.00 \times 10^{-2} \mathrm{e} / \mathrm{nm}^{2}\right.$ in $\mathrm{KCl}$ solution at a concentration of $\left.3 \times 10^{-4} \mathrm{M}\right)$.

Similar to the case of the $\mathrm{CH}_{3}-\mathrm{SAM}$, the OH-SAMs exhibited attraction in air (Fig. 2). This attraction was far beyond the range of the van der Waals interactions, as shown in Fig. 2. To elucidate the origin of the attraction, we investigated the formation of a water layer using quartz crystal microbalance (QCM). The QCM measurements revealed that water layers with a thickness of at least 20 
TABLE I: Values of the parameters obtained in the DLVO fits of the experimental results.

\begin{tabular}{lll}
\hline \hline Monolayer & Decay length $\lambda_{D}(\mathrm{~nm})$ & $\begin{array}{l}\text { Estimated surface excess charge } \\
\text { densities }\left(n_{e x}^{i} / \mathrm{nm}^{-2}\right)\end{array}$ \\
\hline & & \\
$\mathrm{CH}_{3}$-SAM (Fig. 1) & 18 & $1.7 \times 10^{-2}$ \\
OEG-SAM (Fig. 3(a)) & 15 & $0.6 \times 10^{-2}$ \\
\hline \hline
\end{tabular}

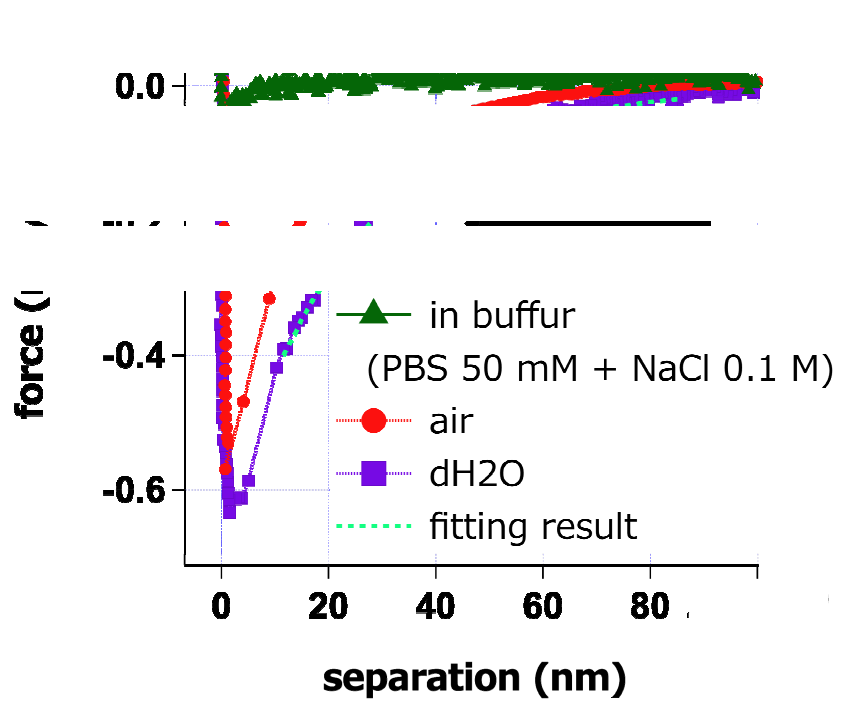

FIG. 2: Force vs distance curves recorded on the approach of an OH-SAM probe to an OH-SAM substrate in air, pure water, and buffer solution (PBS buffur $\mathrm{pH} 7.4,10 \mathrm{mM}$ containing $\mathrm{NaCl}$ at a concentration of $0.6 \mathrm{M})$. The curve measured in pure water was fitted to a single exponential function (dashed line).

$\mathrm{nm}$ were formed on the hydrophilic OH- and OEG-SAMs in ambient environment (temperature and relative humidity in our experimental room are $25^{\circ} \mathrm{C}$ and $50 \%$, respectively). Moreover, these attractions critically depended on the humidity in the experimental room, whereas the curves observed in aqueous solution were highly reproduced. Therefore, we concluded that the attraction is due to the bridging of water formed between the probe and substrate.

A long-range attraction between the OH-SAMs was observed in pure water. At higher ion concentrations, the attraction disappeared. The sensitivity of the attraction to solution conditions indicates an electrostatic origin of the attraction. Meyer et al. reported that dipole moments in parallel with a surface induce long-range attraction that is greater than van der Waals interaction and that the force-distance profile of the attraction is well fitted to an exponential function [25]. The attractive interaction observed by us also obeyed exponential decay. With regard to the structure of the OH-SAM, Poirier et al. reported the crystalline structure of the SAM of mercaptohexanol using a scanning tunneling microscope (STM) in vacuum. The SAM is comprised of patches of single crystalline domain (about $100-200 \mathrm{~nm}^{2}$ ) with different orientational and translational configurations [26]. The authors also found that the terminal groups (hydroxyl groups) strongly interact with each other and each domain is expected to possess a large lateral dipole moment due to the alignment of the hydroxyl groups in the domain. Combining these facts, we expect that this attraction is due to the interaction between lateral dipoles emerged on the probe and substrate.

The important finding here is that there was no repulsion assigned to electrostatic double layer force in pure water, implying that the hydroxyl ions did not tend to accumulate at the interface of the OH-SAM and bulk water. However, Chan et al. previously reported that the OH-SAM bears negative charges at $\mathrm{pH} 6.0$ [23], which seems to contradict the above finding. Unfortunately, the reason for this apparent discrepancy has not been clear. We speculate that the discrepancy is due to the difference in the mobility (stability) of the ions i.e., in the case with the OH-SAM, hydroxide ions are easy to move out from the confined region due to the electrostatic repulsion induced by themselves, when its separation is less than 60 $\mathrm{nm}$. This is contrary to the case of the $\mathrm{CH}_{3}$-SAM.

Next we switch our discussion to the cases of OEGSAMs. The OEG-SAMs both on Au and Ag substrates showed attraction in air. According to the same discussion as in the case of the OH-SAM, we assigned these attractions as capillary force. These monolayers however induced completely different interactions in aqueous solutions. In the case of the Au-supported SAM, strong repulsion was observed in pure water. This repulsion disappeared in buffer solution, indicating that the origin of the repulsion is electrostatic interaction and that charges were accumulated at the SAM-water interface. As shown in Fig. 3(b), attraction was observed between the OEG-SAM and $\mathrm{NH}_{2}$-SAM, which bears positive charge in pure water, indicating that the Au-supported OEGSAM is negatively charged. This result is consistent with the reports by Chan et al. and Dicke et al. in which hydroxide ions were concentrated at the interface of the Au-supported OEG-SAM and water [8, 23].

It is striking that the interactions between $\mathrm{Ag}$ supported SAMs were completely different from those between the Au-supported OEG-SAMs, although both $\mathrm{Au}$ and $\mathrm{Ag}$ substrates were covered with equivalent molecules (Fig. 3(c)). In pure water, no long-range repulsion was observed between Ag-supported OEG-SAMs. The absence of the repulsion indicates the absence of stabilized ions at the SAM-water interface at small (less than $50 \mathrm{~nm}$ ) separations. The main difference between these SAMs is the density of the thiolate molecules in the monolayer. Herrwerth et al. reported that the density of the molecule is about $10 \%$ higher on $\mathrm{Ag}$ than on $\mathrm{Au}$ (the densities of the thiolate molecules per $1 \mathrm{~nm}^{2}$ on $\mathrm{Au}$ and $\mathrm{Ag}$ are 3.79 and 4.19 , respectively) [16]. As found in the results of computer simulations by Pertsin et al., the molecular density critically affects the penetration of water molecules into 

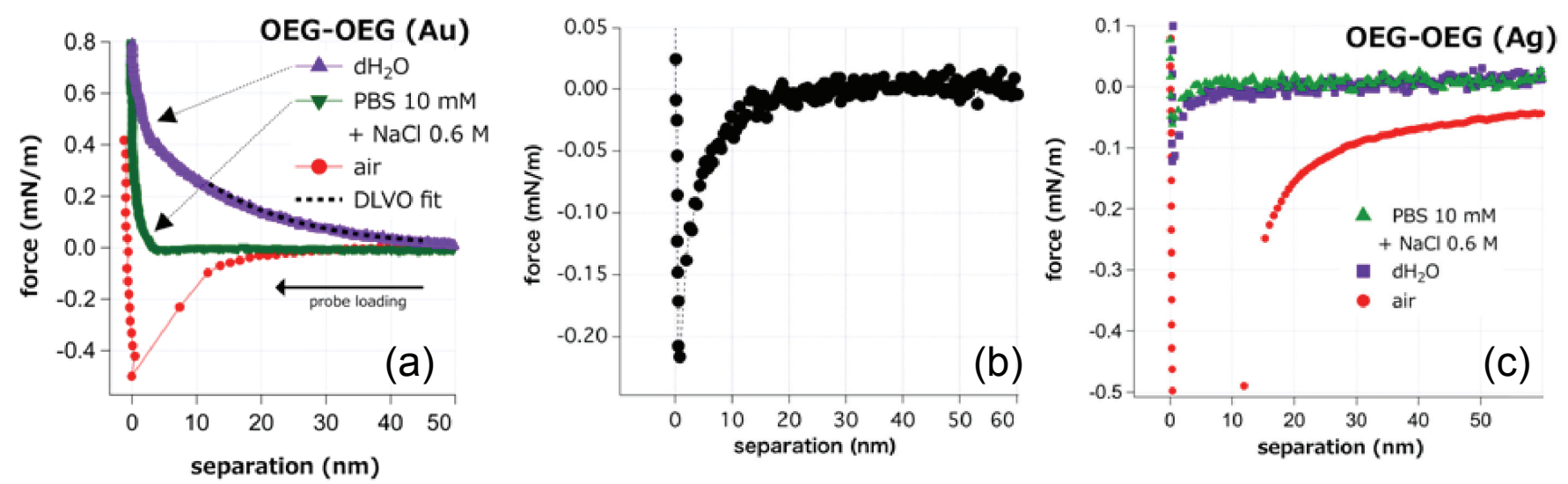

FIG. 3: (a) Force vs distance curves recorded on the approach for the interaction between Au-supported OEG-SAMs in air, pure water, and buffer solution (PBS buffur $\mathrm{pH} 7.4,10 \mathrm{mM}$ containing $\mathrm{NaCl}$ at a concentration of $0.6 \mathrm{M}$ ) The results of the DLVO fitting was indicated with a dashed line. (b) Force vs distance curves recorded on the approach for the interaction between the $\mathrm{Au}$-supported OEG- and $\mathrm{NH}_{2}-\mathrm{SAMs}$ in pure water (c) Force vs distance curves recorded on the approach for the interaction between Ag-supported OEG-SAMs. The experimental conditions are same as those in the case of the Au-supported SAM.

the SAM. In other words, the Au-supported SAM allows water to penetrate into the OEG chains, whereas the Agsupported SAM does not [27]. In the bulk state, the viscosity of water and ethylene glycol or poly(ethylene glycol) is 20-30 times higher than that of bulk water [28, 29]. We expect that the mixing of water with the OEG chains increases the viscosity of the interfacial water and significantly affects the structure or dynamics of the interfacial water, resulting in the stabilization of the hydroxide ions at the interface. On the other hand, the Ag-supported OEG-SAM showed qualitatively similar interactions with OH-SAM, resulting in the similar interfacial behavior of hydroxide ions. Namely, the dynamics or structure of the interfacial water may significantly affect the stability of the surface charges.

\section{CONCLUSION}

We here studied the interfacial behavior of the hydroxide ions in the vicinity of various SAMs. The hydrophobic $\mathrm{CH}_{3}$-SAM induced the accumulation of hydroxide ions at the interface, whereas the hydrophilic OH-SAM did not. This implies that the terminal group, which may significantly affect the structure and ordering of the in- terfacial water molecules, is a critical factor for determining the distribution of the interfacial ions. In the case of the OEG-SAM, the congregation of the hydroxide ions strongly depended on the molecular density of the thiolate molecules constituting SAM. Combining the results of previous computer simulations and our findings, it appears that several factors, such as terminal groups and surface density, govern the accumulation of surface charges. We also found that the effect of surfaceto-surface separations may also be an important factor, because the stability of the loosely adsorbed ions is not so high compared with ions strongly bound to charged surface groups. Therefore, the prediction of the long-range electrostatic interaction between neutral objects based on the result of electrokinetic measurements should be performed with certain care.

\section{Acknowledgments}

Part of this work was supported by a Grant-in-Aid for Young Scientists (B) from MEXT (Ministry of Education, Culture, Sports, Science and Technology).
[1] D. Leckband and J. Israelachvili, Q. Rev. Biophys. 34, 105 (2001).

[2] K. G. Marinova, R. G. Alargova, N. D. Denkov, O. D. Velev, D. N. Petsev, I. B. Ivanov, and R. P. Borwankar, Langmuir 12, 2045 (1996).

[3] R. M. Pashley, J. Phys. Chem. B 107, 1714 (2003).

[4] P. Weidenhammer and H. J. Jacobasch, J. Colloid Interface Sci. 180, 232 (1996).

[5] R. Zimmermann, T. Osaki, T. Kratzmuller, G. Gauglitz, S. S. Dukhin, and C. Werner, Anal. Chem. 78, 5851 (2006).

[6] R. Zangi and J. B. F. N. Engberts, J. Am. Chem. Soc. 127, 2272 (2005).
[7] R. Vacha, R. Zangi, J. B. F. N. Engberts, and P. Jungwirth, J. Phys. Chem. C 112, 7689 (2008).

[8] C. Dicke and G. Hahner, J. Am. Chem. Soc. 124, 12619 (2002).

[9] C. Dicke and G. Hahner, J. Phys. Chem. B 106, 4450 (2002).

[10] R. Schweiss, P. B. Welzel, C. Werner, and W. Knoll, Colloids Surf. A 195, 97 (2001).

[11] F. Schreiber, Prog. Surf. Sci. 65, 151 (2000).

[12] J. C. Love, L. A. Estroff, J. K. Kriebel, R. G. Nuzzo, and G. M. Whitesides, Chem. Rev. 105, 1103 (2005).

[13] K. L. Prime and G. M. Whitesides, Science 252, 1164 (1991). 
[14] K. L. Prime and G. M. Whitesides, J. Am. Chem. Soc. 115, 10714 (1993).

[15] J. L. Hutter and J. Bechhoefer, Rev. Sci. Instrum. 64, 1868 (1993).

[16] S. Herrwerth, W. Eck, S. Reinhardt, and M. Grunze, J. Am. Chem. Soc. 125, 9359 (2003).

[17] J. Israelachvili, Intermolecular and Surface Forces (Academic Press, London, 1992).

[18] H. J. Butt, B. Cappella, and M. Kappl, Surf. Sci. Rep. 59, 1 (2005).

[19] S. P. Jarvis and J. B. Pethica, Thin Solid Films 273, 284 (1996).

[20] A. V. Nguyen, J. Nalaskowski, J. D. Miller, and H. J. Butt, Int. J. Miner. Process. 72, 215 (2003).

[21] K. Lum, D. Chandler, and J. D. Weeks, J. Phys. Chem. B 103, 4570 (1999).

[22] T. Hayashi, A. J. Pertsin, and M. Grunze, J. Chem. Phys.
117, 6271 (2002).

[23] Y. H. M. Chan, R. Schweiss, C. Werner, and M. Grunze, Langmuir 19, 7380 (2003).

[24] R. Schweiss, P. B. Welzel, C. Werner, and W. Knoll, Langmuir 17, 4304 (2001)

[25] E. E. Meyer, Q. Lin, T. Hassenkam, E. Oroudjev, and J. N. Israelachvili, Proc. Natl. Acad. Sci. USA 102, 6839 (2005).

[26] G. E. Poirier, E. D. Pylant, and J. M. White, J. Chem. Phys. 105, 2089 (1996).

[27] A. J. Pertsin, T. Hayashi, and M. Grunze, J. Phys. Chem. B 106, 12274 (2002).

[28] A. Pal and Y. P. Singh, J. Chem. Eng. Data 41, 1008 (1996).

[29] T. M. Aminabhavi and B. Gopalakrishna, J. Chem. Eng. Data 40, 856 (1995). 